\title{
Facile $N$-Arylation of Amines and Sulfonamides
}

\author{
Zhijian Liu and Richard C. Larock * \\ Department of Chemistry, Iowa State University, Ames, Iowa 50011
}

\section{Supporting Information}

General. The ${ }^{1} \mathrm{H}$ and ${ }^{13} \mathrm{C}$ NMR spectra were recorded at 300 and $75.5 \mathrm{MHz}$ or 400 and $100 \mathrm{MHz}$ respectively. All melting points are uncorrected. High resolution mass spectra were recorded on a Kratos MS50TC double focusing magnetic sector mass spectrometer using EI at $70 \mathrm{eV}$. All reagents were used directly as obtained commercially unless otherwise noted. All yields reported in the publication represent an average of at least two independent runs. CsF and acetonitrile were purchased from Sigma-aldrich Co.. The methoxy-substituted silylaryl triflate $\mathbf{1 b}$ was prepared according to a previous literature procedure. ${ }^{1}$

General procedure for the $N$-arylation of amines and sulfonamides.

Procedure A: To a solution of $\mathrm{MeCN}$ (4 mL), amine or sulfonamide $(0.25 \mathrm{mmol})$ and silylaryl triflate $(0.225 \mathrm{mmol})$ was added $\mathrm{CsF}(0.45 \mathrm{mmol})$. The reaction mixture was allowed to stir at room temperature for $1 \mathrm{~d}$ and the resulting solution was washed by brine $(20 \mathrm{~mL})$ and extracted with diethyl ether $(20 \mathrm{~mL})$. The combined ether fractions were dried over $\mathrm{Na}_{2} \mathrm{SO}_{4}$ and concentrated under reduced pressure. The residue was purified by flash chromatography on silica gel to afford the desired product.

Procedure B: To a solution of $\mathrm{MeCN}(4 \mathrm{~mL})$, amine or sulfonamide $(0.25 \mathrm{mmol})$ and silylaryl triflate $(0.6 \mathrm{mmol})$ was added $\mathrm{CsF}(1.2 \mathrm{mmol})$. The reaction mixture was allowed to stir at room temperature for $3 \mathrm{~d}$ for $\mathrm{N}$-arylated amines and $1 \mathrm{~d}$ for $\mathrm{N}$-arylated sulfonamides. The resulting solution was washed with brine $(20 \mathrm{~mL})$ and extracted with diethyl ether $(20 \mathrm{~mL})$. The combined ether fractions were dried over $\mathrm{Na}_{2} \mathrm{SO}_{4}$ and concentrated under reduced pressure. The residue was purified by flash chromatography on silica gel to afford the desired product. 
Diphenylamine (entry 1, Table 1). Using procedure A, silylaryl triflate $1 \mathrm{a}(0.225 \mathrm{mmol}$, $67 \mathrm{mg})$, aniline $(0.25 \mathrm{mmol}, 24 \mathrm{mg})$ and $\mathrm{CsF}(0.45 \mathrm{mmol}, 69 \mathrm{mg})$ afforded the indicated compound $\left(31 \mathrm{mg}\right.$ ) in an $81 \%$ yield as a white solid: $\mathrm{mp} 49-50{ }^{\circ} \mathrm{C}$ (lit. ${ }^{2} \mathrm{mp} 50-52{ }^{\circ} \mathrm{C}$ ). The ${ }^{1} \mathrm{H}$ and ${ }^{13} \mathrm{C}$ NMR spectra match the literature data. ${ }^{3}{ }^{1} \mathrm{H}$ NMR (400 MHz, $\left.\mathrm{CDCl}_{3}\right) \delta$ $7.26(\mathrm{td}, J=8.0,1.2 \mathrm{~Hz}, 4 \mathrm{H}), 7.05(\mathrm{dd}, J=8.0,1.2 \mathrm{~Hz}, 4 \mathrm{H}), 6.93(\mathrm{td}, J=8.0,1.2 \mathrm{~Hz}$, 2H), 5.61 (br s, $1 \mathrm{H}) ;{ }^{13} \mathrm{C} \mathrm{NMR}\left(100 \mathrm{MHz}, \mathrm{CDCl}_{3}\right) \delta 143.6$ (2C), 129.8 (4C), 121.4 (2C), 118.3 (4C).

$\mathrm{N}$-Phenyl-3-methoxyaniline (entry 2, Table 1). Using procedure A, silylaryl triflate $\mathbf{1 b}$ $(0.225 \mathrm{mmol}, 74 \mathrm{mg})$, aniline $(0.25 \mathrm{mmol}, 24 \mathrm{mg})$ and $\mathrm{CsF}(0.45 \mathrm{mmol}, 69 \mathrm{mg})$ afforded the indicated compound (38 mg) in an $84 \%$ yield as a colorless oil. The ${ }^{1} \mathrm{H}$ and ${ }^{13} \mathrm{C}$ NMR spectra match the literature data. ${ }^{4}{ }^{1} \mathrm{H}$ NMR $\left(300 \mathrm{MHz}, \mathrm{CDCl}_{3}\right) \delta 7.33-7.10(\mathrm{~m}, 5 \mathrm{H}), 6.97$ (t, $J=7.4 \mathrm{~Hz}, 1 \mathrm{H}), 6.69-6.67$ (m, 2H), 6.51 (dd, $J=7.4,1.9 \mathrm{~Hz}, 1 \mathrm{H}), 5.74(\mathrm{~s}, 1 \mathrm{H}), 3.80$ (s, 3H); ${ }^{13} \mathrm{C}$ NMR (75 MHz, CDCl $) \delta 160.8$ (1C), 144.7 (1C), 142.9 (1C), 130.2 (1C), 129.5 (1C), 121.4 (1C), 118.5 (1C), 110.4 (1C), 106.3 (1C), 103.4 (1C), 55.4 (1C).

Triphenylamine (entry 3, Table 1). Using procedure B, aniline ( $0.25 \mathrm{mmol}, 24 \mathrm{mg})$, silylaryl triflate $1 \mathrm{a}(0.6 \mathrm{mmol}, 179 \mathrm{mg})$ and $\mathrm{CsF}(1.2 \mathrm{mmol}, 182 \mathrm{mg})$ afforded the indicated compound $(60 \mathrm{mg})$ in a $98 \%$ yield as a white solid: $\mathrm{mp} 123-124{ }^{\circ} \mathrm{C}\left(\mathrm{lit} .{ }^{5} \mathrm{mp}\right.$ 125-126 ${ }^{\circ} \mathrm{C}$ ). The ${ }^{1} \mathrm{H}$ and ${ }^{13} \mathrm{C}$ NMR spectra match the literature data. ${ }^{6}{ }^{1} \mathrm{H}$ NMR (300 $\left.\mathrm{MHz}, \mathrm{CDCl}_{3}\right) \delta$ 7.43-7.29 (m, 6H), 7.18-7.15 (m, 6H), 7.11-7.04 (m, 3H); ${ }^{13} \mathrm{C}$ NMR (75 $\left.\mathrm{MHz}, \mathrm{CDCl}_{3}\right) \delta 148.2$ (3C), 129.5 (6C), 124.5 (6C), 123.0 (3C).

$\mathbf{N}, \mathbf{N}$-bis(3-Methoxyphenyl)aniline (entry 4, Table 1). Using procedure $\mathrm{B}$, aniline $(0.25$ mmol, $24 \mathrm{mg})$, silylaryl triflate $\mathbf{1 b}(0.6 \mathrm{mmol}, 196 \mathrm{mg})$ and CsF (1.2 mmol, $182 \mathrm{mg})$ afforded the indicated compound $(72 \mathrm{mg})$ in a $94 \%$ yield as a colorless oil. ${ }^{1} \mathrm{H}$ NMR (300 MHz, $\left.\mathrm{CDCl}_{3}\right) \delta$ 7.26-7.21 (m, 2H), 7.16-7.08 (m, 4H), 7.03-6.98 (m, 1H), 6.68-6.62 $(\mathrm{m}, 4 \mathrm{H}), 6.57-6.54(\mathrm{dd}, J=8.1,0.9 \mathrm{~Hz}, 2 \mathrm{H}), 3.71(\mathrm{~s}, 6 \mathrm{H}) ;{ }^{13} \mathrm{C} \mathrm{NMR}\left(75 \mathrm{MHz}, \mathrm{CDCl}_{3}\right) \delta$ 160.6 (2C), 149.2 (2C), 147.8 (1C), 129.9 (2C), 129.4 (2C), 124.8 (2C), 123.2 (1C), 116.9 (2C), 110.2 (2C), 108.4 (2C), 55.4 (2C). IR $\left(\mathrm{CDCl}_{3}\right) 3000,2953 \mathrm{~cm}^{-1} ;$ HRMS m/z 305.1428 (calcd $\mathrm{C}_{20} \mathrm{H}_{19} \mathrm{NO}_{2}, 305.1415$ ).

$\mathrm{N}$-Phenyl-2-iodoaniline (entry 5, Table 1). Using procedure A, silylaryl triflate 1a $(0.225 \mathrm{mmol}, 67 \mathrm{mg}), o$-iodoaniline $(0.25 \mathrm{mmol}, 55 \mathrm{mg})$ and $\mathrm{CsF}(0.45 \mathrm{mmol}, 69 \mathrm{mg})$ afforded the indicated compound $\left(55 \mathrm{mg}\right.$ ) in an $83 \%$ yield as a light yellow oil. ${ }^{1} \mathrm{H}$ NMR 
(300 MHz, $\left.\mathrm{CDCl}_{3}\right) \delta$ 7.78-7.75 (m, 1H), 7.34-7.28 (m, 2H), 7.20-7.18 (m, 2H), 7.14-7.10 (m, 2H), 7.05-7.00 (m, 1H), 6.64-6.58 (m, 1H), $5.90(\mathrm{~s}, 1 \mathrm{H}) ;{ }^{13} \mathrm{C} \mathrm{NMR}\left(75 \mathrm{MHz}, \mathrm{CDCl}_{3}\right)$ $\delta 144.2$ (1C), 142.2 (1C), 139.7 (1C), 129.7 (1C), 129.3 (1C), 122.8 (1C), 122.1 (1C), 120.2 (1C), 116.1 (1C), 89.0 (1C). IR (CDCl $) 3379,3041 \mathrm{~cm}^{-1}$; HRMS m/z 294.9865 (calcd $\mathrm{C}_{12} \mathrm{H}_{10} \mathrm{IN}, 294.9858$ ).

$N$-Propargylaniline (entry 6, Table 1). Using procedure A, silylaryl triflate 1a $(0.225$ mmol, $67 \mathrm{mg})$, propargyl amine $(0.25 \mathrm{mmol}, 14 \mathrm{mg})$ and CsF $(0.45 \mathrm{mmol}, 69 \mathrm{mg})$ afforded the indicated compound $(19 \mathrm{mg})$ in a $62 \%$ yield as a light yellow oil. The ${ }^{1} \mathrm{H}$ NMR spectrum matchs the literature data. ${ }^{7} \mathrm{H}$ NMR $\left(300 \mathrm{MHz}, \mathrm{CDCl}_{3}\right) \delta$ 7.25-7.18 (m, $2 \mathrm{H}), 6.81-6.72(\mathrm{~m}, 1 \mathrm{H}), 6.70-6.67(\mathrm{~m}, 2 \mathrm{H}), 3.94(\mathrm{~m}, 3 \mathrm{H}), 2.12(\mathrm{t}, J=2.4 \mathrm{~Hz}, 1 \mathrm{H}) ;{ }^{13} \mathrm{C}$ NMR (75 MHz, $\left.\mathrm{CDCl}_{3}\right) \delta 147.1$ (1C), 129.4 (2C), 118.9 (1C), 113.7 (2C), 81.2 (1C), 71.5 (1C), 33.9 (1C).

$N$-Phenyl- $N$-propargylaniline (entry 7, Table 1). Using procedure B, propargyl amine $(0.25 \mathrm{mmol}, 14 \mathrm{mg})$, silylaryl triflate $1 \mathrm{a}(0.6 \mathrm{mmol}, 179 \mathrm{mg})$ and CsF $(1.2 \mathrm{mmol}, 182$ $\mathrm{mg}$ ) afforded the indicated compound $(40 \mathrm{mg})$ in a $78 \%$ yield as a light yellow oil. ${ }^{1} \mathrm{H}$ NMR (300 MHz, $\left.\mathrm{CDCl}_{3}\right) \delta$ 7.32-7.23 (m, 4H), 7.10-6.98 (m, 6H), 4.41 (d, $J=2.4 \mathrm{~Hz}$, $2 \mathrm{H}), 2.22(\mathrm{t}, J=2.4 \mathrm{~Hz}, 1 \mathrm{H}) ;{ }^{13} \mathrm{C} \mathrm{NMR}\left(75 \mathrm{MHz}, \mathrm{CDCl}_{3}\right) \delta 147.6(2 \mathrm{C}), 129.5(2 \mathrm{C})$, 122.4 (2C), 121.4 (2C), 80.1 (1C), 72.5 (1C), 42.3 (1C). IR ( $\left.\mathrm{CDCl}_{3}\right)$ 3289, 3063, 2918 $\mathrm{cm}^{-1}$; HRMS m/z 207.1051 (calcd $\mathrm{C}_{15} \mathrm{H}_{13} \mathrm{~N}, 207.1048$ ).

$N$-(2-Cyclohex-1-en-1-ylethyl)- $N, N$-diphenylamine (entry 8, Table 1). Using procedure B, 2-(cyclohex-1-enyl)ethyl amine $(0.25 \mathrm{mmol}, 31 \mathrm{mg})$, silylaryl triflate 1a $(0.6 \mathrm{mmol}, 179 \mathrm{mg})$ and $\mathrm{CsF}(1.2 \mathrm{mmol}, 182 \mathrm{mg})$ afforded the indicated compound (69 $\mathrm{mg})$ in a $99 \%$ yield as a colorless oil. ${ }^{1} \mathrm{H}$ NMR $\left(300 \mathrm{MHz}, \mathrm{CDCl}_{3}\right) \delta 7.28-7.21(\mathrm{~m}, 4 \mathrm{H})$, 7.00-6.89 (m, 6H), 5.44 (s, 1H), 3.80-3.75 (m, 2H), 2.31-2.25 (t, $J=1.2 \mathrm{~Hz}, 2 \mathrm{H}), 1.97-$ $1.92(\mathrm{~m}, 4 \mathrm{H}), 1.60-1.51(\mathrm{~m}, 4 \mathrm{H}) ;{ }^{13} \mathrm{C}$ NMR $\left(75 \mathrm{MHz}, \mathrm{CDCl}_{3}\right) \delta 148.1(2 \mathrm{C}), 135.5(1 \mathrm{C})$, 129.4 (2C), 123.0 (1C), 121.2 (2C), 121.1 (2C), 51.4 (1C), 35.8 (1C), 28.8 (1C), 25.5 (1C), 23.1 (1C), 22.6 (1C). IR ( $\left.\mathrm{CDCl}_{3}\right) 3057,3034,2925 \mathrm{~cm}^{-1}$; HRMS m/z 277.1835 (calcd $\mathrm{C}_{20} \mathrm{H}_{23} \mathrm{~N}, 277.1830$ ).

3-Diphenylamino-1-propanol (entry 9, Table 1). Using procedure B, 3-amino-1propanol $(0.25 \mathrm{mmol}, 19 \mathrm{mg})$, silylaryl triflate $1 \mathrm{a}(0.6 \mathrm{mmol}, 179 \mathrm{mg})$ and $\mathrm{CsF}(1.2$ $\mathrm{mmol}, 182 \mathrm{mg}$ ) afforded the indicated compound $(47 \mathrm{mg})$ in an $83 \%$ yield as a colorless 
oil. ${ }^{1} \mathrm{H}$ NMR $\left(300 \mathrm{MHz}, \mathrm{CDCl}_{3}\right) \delta$ 7.27-7.20 (m, 4H), 7.02-6.89 (m, 6H), $3.31(\mathrm{t}, J=7.2$ $\mathrm{Hz}, 2 \mathrm{H}), 3.68(\mathrm{t}, J=6.0 \mathrm{~Hz}, 2 \mathrm{H}), 1.92-1.83(\mathrm{~m}, 2 \mathrm{H}), 1.73(\mathrm{~s}, 1 \mathrm{H}) ;{ }^{13} \mathrm{C}$ NMR $(75 \mathrm{MHz}$, $\left.\mathrm{CDCl}_{3}\right) \delta 148.3$ (2C), 129.5 (2C), 121.6 (1C), 121.3 (2C), 60.8 (1C), 49.3 (1C), 30.6 (1C). IR $\left(\mathrm{CDCl}_{3}\right) 3351,3041,2943 \mathrm{~cm}^{-1} ; \mathrm{HRMS} \mathrm{m} / \mathrm{z} 227.1316$ (calcd $\mathrm{C}_{15} \mathrm{H}_{17} \mathrm{NO}$, 227.1310).

$N$-tert-Butyl- $N$-phenylaniline (entry 10, Table 1). Using procedure B, tert-butylamine (0.25 mmol, $18.5 \mathrm{mg})$, silylaryl triflate $1 \mathbf{a}(0.6 \mathrm{mmol}, 179 \mathrm{mg})$ and CsF (1.2 mmol, 182 $\mathrm{mg}$ ) afforded the indicated compound $(55 \mathrm{mg})$ in a $97 \%$ yield as a colorless oil. The ${ }^{1} \mathrm{H}$ and ${ }^{13} \mathrm{C}$ NMR spectra match the literature data. ${ }^{8}{ }^{1} \mathrm{H}$ NMR $\left(300 \mathrm{MHz}, \mathrm{CDCl}_{3}\right) \delta 7.20-6.94$ (m, 10H), 1.40 (s, 9H); ${ }^{13} \mathrm{C}$ NMR (75 MHz, $\left.\mathrm{CDCl}_{3}\right) \delta 148.9$ (2C), 128.9 (2C), 127.1 (2C), 122.5 (2C), 56.0 (1C), 30.5 (3C).

$N$-Methyl- $N$-phenylaniline (entry 11, Table 1). Using procedure B, $N$-methylaniline (0.25 mmol, $27 \mathrm{mg})$, silylaryl triflate $1 \mathbf{a}(0.3 \mathrm{mmol}, 90 \mathrm{mg})$ and $\mathrm{CsF}(0.6 \mathrm{mmol}, 91 \mathrm{mg})$ afforded the indicated compound $(66 \mathrm{mg})$ in a $93 \%$ yield as a colorless oil. The ${ }^{1} \mathrm{H}$ and ${ }^{13} \mathrm{C}$ NMR spectra match the literature data. ${ }^{9} \mathrm{H}$ NMR $\left(300 \mathrm{MHz}, \mathrm{CDCl}_{3}\right) \delta 7.33-7.23(\mathrm{~m}$, 4H), 7.08-6.94 (m, 6H), 3.34 (s, 3H); ${ }^{13} \mathrm{C}$ NMR (75 MHz, $\left.\mathrm{CDCl}_{3}\right) \delta 149.0(2 \mathrm{C}), 129.2$ (2C), 121.2 (2C), 120.4 (2C), 40.2 (1C).

$N, N$-Diphenylmethanesulfonamide (entry 12, Table 1). Using procedure B, methanesulfonamide $(0.25 \mathrm{mmol}, 24 \mathrm{mg})$, silylaryl triflate $1 \mathbf{a}(0.6 \mathrm{mmol}, 179 \mathrm{mg})$ and $\mathrm{CsF}(1.2 \mathrm{mmol}, 182 \mathrm{mg}$ ) afforded the indicated compound $(50 \mathrm{mg})$ in an $80 \%$ yield as a white solid: $\mathrm{mp} 115-117{ }^{\circ} \mathrm{C}$; ${ }^{1} \mathrm{H}$ NMR (400 MHz, $\left.\mathrm{CDCl}_{3}\right) \delta 7.41-7.35$ (m, 8H), 7.31-7.25 (m, 2H), 3.16 (s, 3H); ${ }^{13} \mathrm{C}$ NMR (100 MHz, $\left.\mathrm{CDCl}_{3}\right) \delta 141.5$ (2C), 129.7 (2C), 127.8 (2C), 127.5 (2C), 40.3 (1C). IR ( $\left.\mathrm{CDCl}_{3}\right) 3071,2983,1343 \mathrm{~cm}^{-1}$; HRMS m/z 247.0672 (calcd $\left.\mathrm{C}_{13} \mathrm{H}_{13} \mathrm{NO}_{2} \mathrm{~S}, 247.0667\right)$.

$N, N$-Diphenylbenzenesulfonamide (entry 13, Table 1). Using procedure B, benzenesulfonamide $(0.25 \mathrm{mmol}, 39 \mathrm{mg})$, silylaryl triflate $1 \mathbf{a}(0.6 \mathrm{mmol}, 179 \mathrm{mg})$ and CsF (1.2 mmol, $182 \mathrm{mg}$ ) afforded the indicated compound $(77 \mathrm{mg})$ in a $99 \%$ yield as a white solid: $\mathrm{mp} 120-121{ }^{\circ} \mathrm{C} ;{ }^{1} \mathrm{H}$ NMR $\left(300 \mathrm{MHz}, \mathrm{CDCl}_{3}\right) \delta 7.73-7.70$ (m, 2H), 7.62-7.45 $(\mathrm{m}, 3 \mathrm{H}), 7.34-7.23(\mathrm{~m}, 10 \mathrm{H}) ;{ }^{13} \mathrm{C} \mathrm{NMR}\left(75 \mathrm{MHz}, \mathrm{CDCl}_{3}\right) \delta 141.6$ (2C), 140.7 (1C), 133.0 (1C), 129.5 (4C), 129.1 (2C), 128.5 (4C), 127.9 (2C), 127.7 (2C). IR ( $\left.\mathrm{CDCl}_{3}\right)$ $3061,1353 \mathrm{~cm}^{-1}$; HRMS m/z 309.0829 (calcd $\mathrm{C}_{18} \mathrm{H}_{15} \mathrm{NO}_{2} \mathrm{~S}, 309.0823$ ). 
N,N-bis(3-Methoxyphenyl)-4-methylbenzenesulfonamide (entry 14, Table 1). Using procedure $\mathrm{B}, p$-toluenesulfonamide $(0.25 \mathrm{mmol}, 43 \mathrm{mg})$, silylaryl triflate $\mathbf{1 b}(0.6 \mathrm{mmol}$, $197 \mathrm{mg}$ ) and CsF (1.2 mmol, $182 \mathrm{mg}$ ) afforded the indicated compound (88 $\mathrm{mg}$ ) in a 92 $\%$ yield as a colorless oil. ${ }^{1} \mathrm{H}$ NMR $\left(300 \mathrm{MHz}, \mathrm{CDCl}_{3}\right) \delta 7.62(\mathrm{~d}, J=8.4 \mathrm{~Hz}, 2 \mathrm{H}), 7.26$ $(\mathrm{d}, J=8.1 \mathrm{~Hz}, 2 \mathrm{H}), 7.22-7.16(\mathrm{~m}, 2 \mathrm{H}), 6.84-6.77(\mathrm{~m}, 6 \mathrm{H}), 3.73(\mathrm{~s}, 6 \mathrm{H}), 2.41(\mathrm{~s}, 3 \mathrm{H}) ;{ }^{13} \mathrm{C}$ NMR (75 MHz, $\left.\mathrm{CDCl}_{3}\right) \delta 160.3$ (2C), 143.9 (1C), 142.7 (2C), 137.8 (1C), 129.9 (2C), 129.7 (2C), 128.0 (2C), 120.5 (2C), 114.4 (2C), 113.2 (2C), 55.6 (2C), 21.8 (1C). IR $\left(\mathrm{CDCl}_{3}\right)$ 3052, 2958, $1354 \mathrm{~cm}^{-1}$; HRMS m/z 383.1199 (calcd $\mathrm{C}_{21} \mathrm{H}_{21} \mathrm{NO}_{4} \mathrm{~S}, 383.1191$ ).

$N$-Methyl- $N$-phenyl-p-toluenesulfonamide (entry 15, Table 1). Using procedure B, $N$ methyl- $p$-toluenesulfonamide $(0.25 \mathrm{mmol}, 47 \mathrm{mg})$, silylaryl triflate $1 \mathrm{a}(0.3 \mathrm{mmol}, 89 \mathrm{mg})$ and $\mathrm{CsF}(0.5 \mathrm{mmol}, 76 \mathrm{mg})$ afforded the indicated compound $(57 \mathrm{mg})$ in an $87 \%$ yield as a white solid: $\mathrm{mp} 92-93{ }^{\circ} \mathrm{C} ;{ }^{1} \mathrm{H}$ NMR $\left(300 \mathrm{MHz}, \mathrm{CDCl}_{3}\right) \delta$ 7.43-7.40 (m, 2H), 7.32-7.21 $(\mathrm{m}, 5 \mathrm{H}), 7.11-7.07(\mathrm{~m}, 2 \mathrm{H}), 3.15(\mathrm{~s}, 3 \mathrm{H}), 2.40(\mathrm{~s}, 3 \mathrm{H}) ;{ }^{13} \mathrm{C} \mathrm{NMR}\left(75 \mathrm{MHz}, \mathrm{CDCl}_{3}\right) \delta$ 143.8 (1C), 141.8 (1C), 133.7 (1C), 129.5 (2C), 129.0 (2C), 128.1 (2C), 127.4 (1C), 126.8 (2C), 38.3 (1C), 21.7 (1C). IR $\left(\mathrm{CDCl}_{3}\right)$ 3018, 2918, $1344 \mathrm{~cm}^{-1} ; \mathrm{HRMS} \mathrm{m} / \mathrm{z}$ 261.0828 (calcd $\mathrm{C}_{14} \mathrm{H}_{15} \mathrm{NO}_{2} \mathrm{~S}, 261.0823$ ).

\section{References}

(1) Pena, D.; Perez, D.; Guitian, E.; Castedo, L. J. Am. Chem. Soc. 1999, 121, 5827.

(2) Desmarets, C.; Schneider, R.; Fort, Y. J. Org. Chem. 2002, 67, 3029.

(3) Kataoka, N.; Shelby, Q.; Stambuli, J. P.; Hartwig, J. F. J. Org. Chem. 2002, 67, 5553.

(4) Driver, M. S.; Hartwig, J. F. J. Am. Chem. Soc. 1996, 118, 7217.

(5) Haga, K.; Oohashi, M.; Kaneko, R. Bull. Chem. Soc. Jpn. 1984, 57, 1586.

(6) Shi, L.; Wang, M.; Fan, Ch.-A.; Zhang, F.-M.; Tu, Y-Q. Org. Lett. 2003, 5, 3515.

(7) Yoshida, Y.; Tanabe, Y. Synthesis 1997, 533.

(8) Prashad, M.; Mak, X. Y.; Liu, Y.; Repič, O. J. Org. Chem. 2003, 68, 1163.

(9) Ishikawa, T.; Uedo, E.; Tani, R.; Saito, S. J. Org. Chem. 2001, 66, 186. 

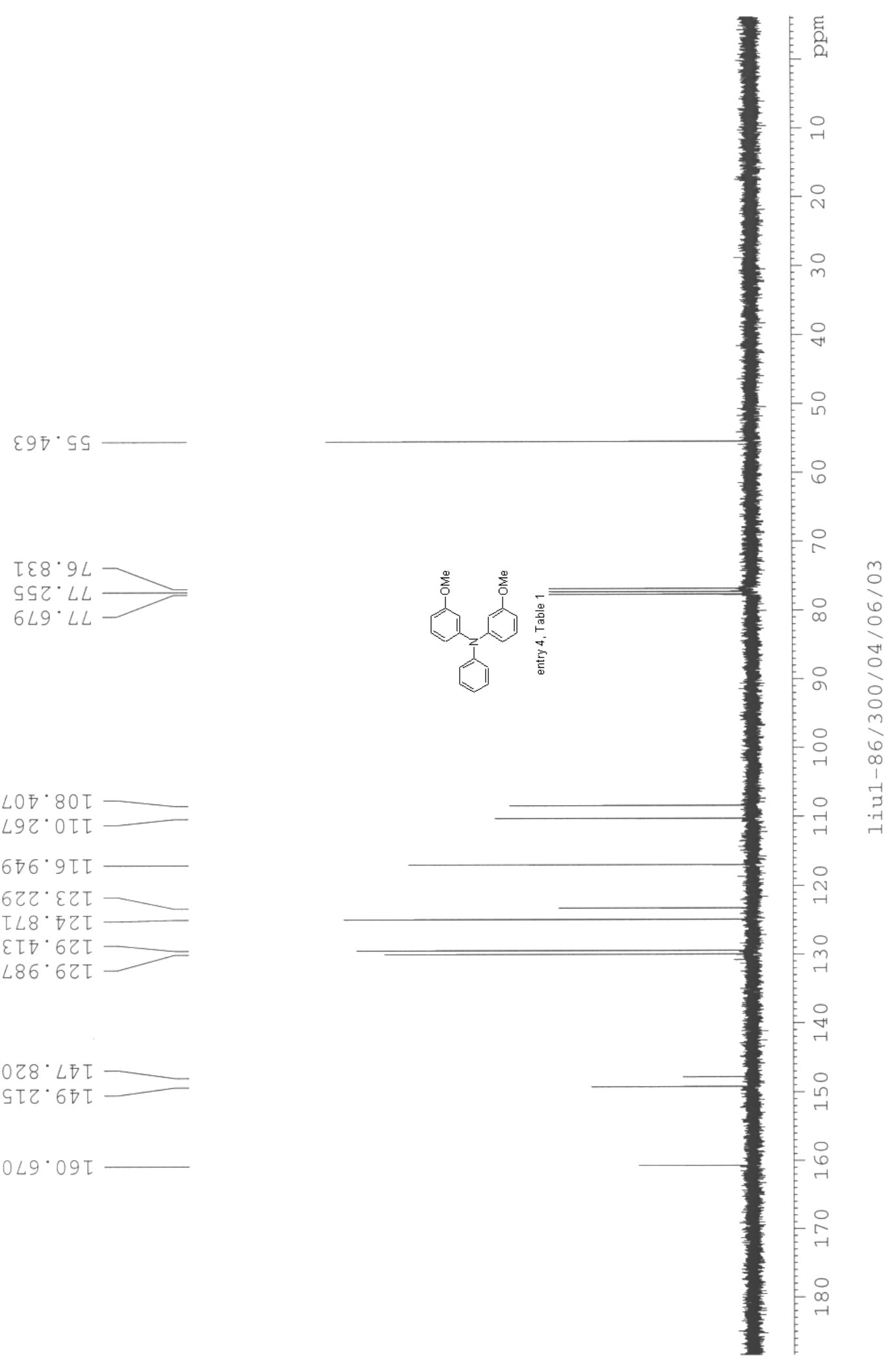

$\angle 07 \cdot 80 T$

L92.0IT

$6 \nabla 6 \cdot 9 \tau \tau$

$6 乙 2 \cdot \varepsilon 乙 T$

โL8'万乙Т

$\varepsilon \tau \nabla \cdot 6 Z \tau$

$486^{\circ} 62 \mathrm{~T}$

$028^{\circ} \angle \bar{L}$

STZ・67t

$0 \angle 9^{\circ} 09 \tau$ 


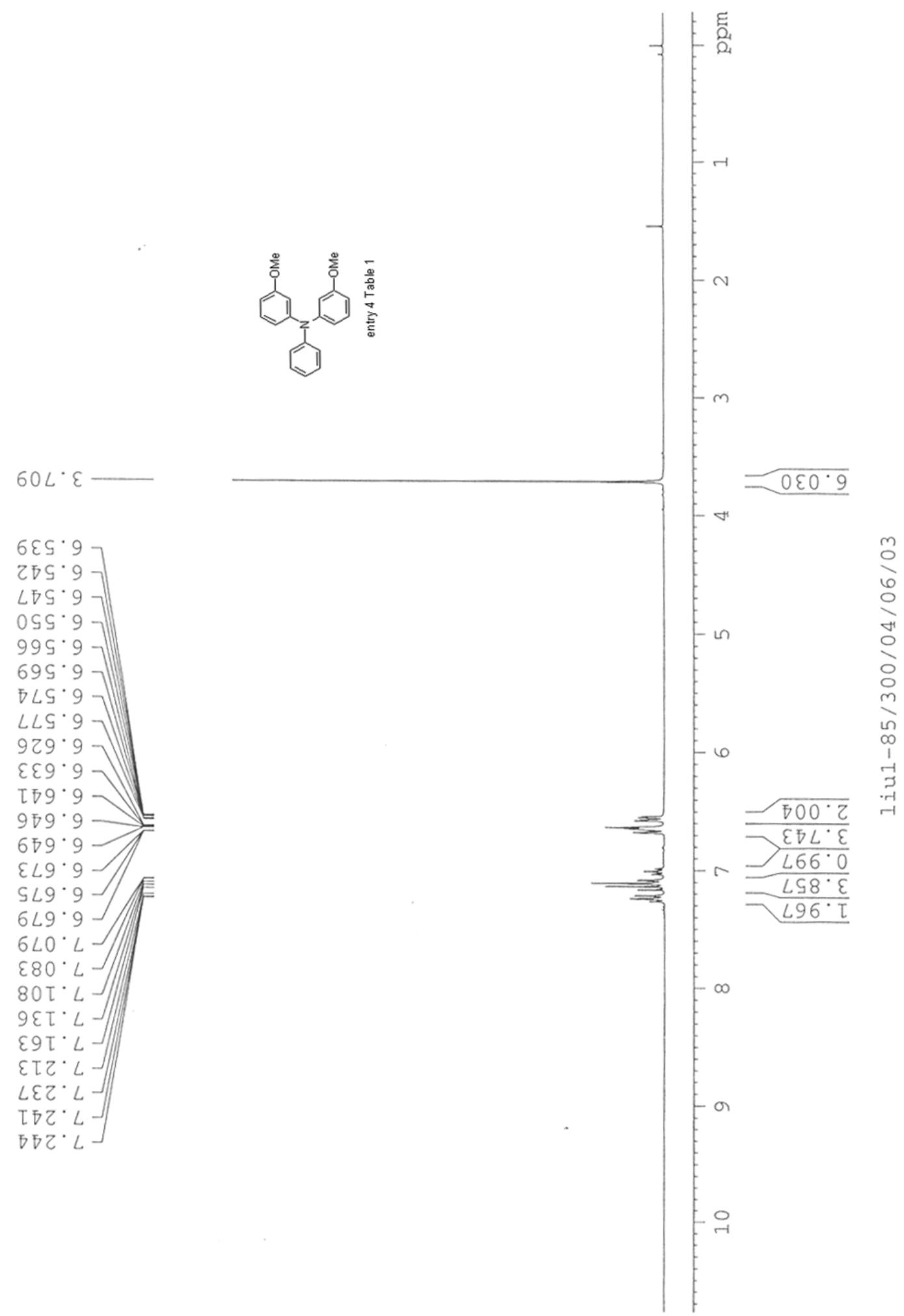




$$
1
$$


$000 \cdot 0-$

$8 \varepsilon S^{\circ} \mathrm{T}$

$206^{\circ} \mathrm{s}-$

$789 \cdot 97$

$969^{\circ} 97$

$209 \cdot 97$

โโ9.9

ع โ9.9

ธ29.9

$079 \cdot 9$

$466^{\circ} 9$

โ00.

$700^{\circ} \mathrm{L}$

$920^{\circ} \mathrm{L}$

$\left.970^{\circ} \mathrm{L}\right]$

OS $0^{\circ}$

¿90

$860^{\circ} L$

$90 \tau^{\circ} L$

$\left.60 \tau^{\circ} L\right]$

LZT.

Ђहโ $\cdot L$

$L \varepsilon \tau \cdot L$

ZST'

S૬โ.

$6 L I \cdot L$

$78 \tau^{\circ}$

$\angle 6 \tau \cdot L$

$66 \tau^{\circ} L$

ZโZ $L]$

$9 \pi 2 \cdot L$

$L L Z \cdot L]$

$\nabla 8 Z^{\circ} L$

$062^{\circ} L$

$80 \varepsilon \cdot L-$

โโ $\varepsilon^{\circ}\llcorner]$

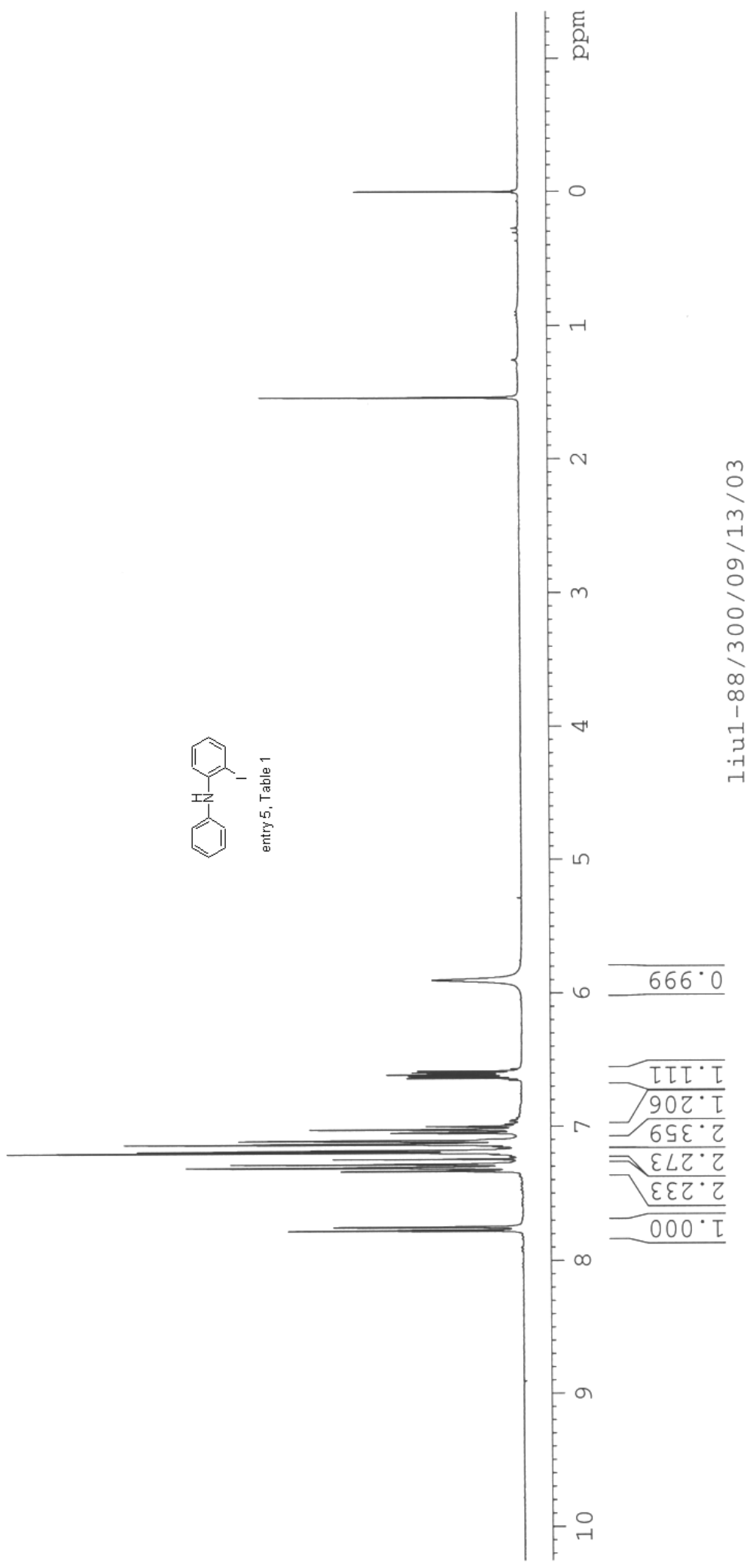




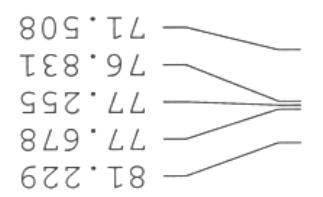

$9 S L \cdot \varepsilon \tau \tau-$

$988 \cdot 8 \tau \tau$

โ8ゅ $62 \tau$

$\angle \angle O^{\circ} \angle D T$

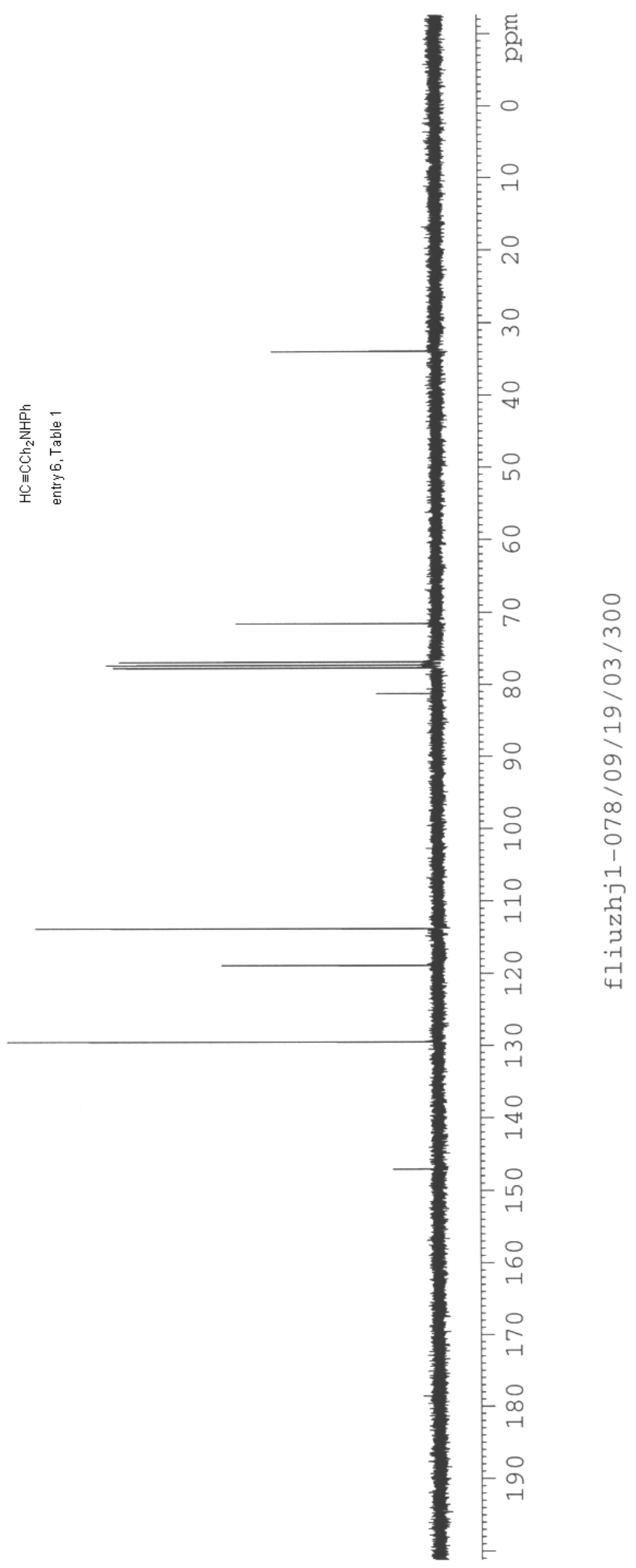


$000^{\circ} 0$

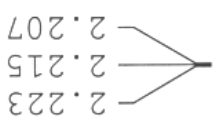

$\varsigma \varepsilon \sigma^{\circ} \varepsilon \longrightarrow$
$\varepsilon \varpi \sigma^{\circ} \varepsilon \longrightarrow$

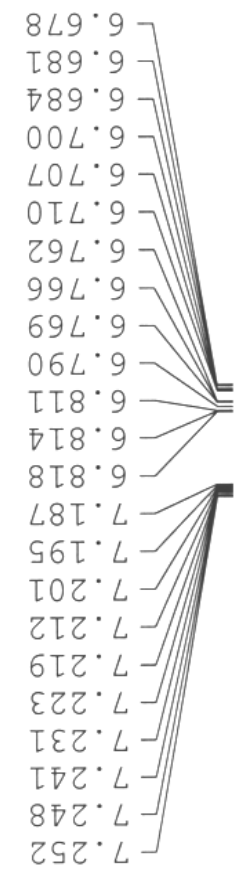

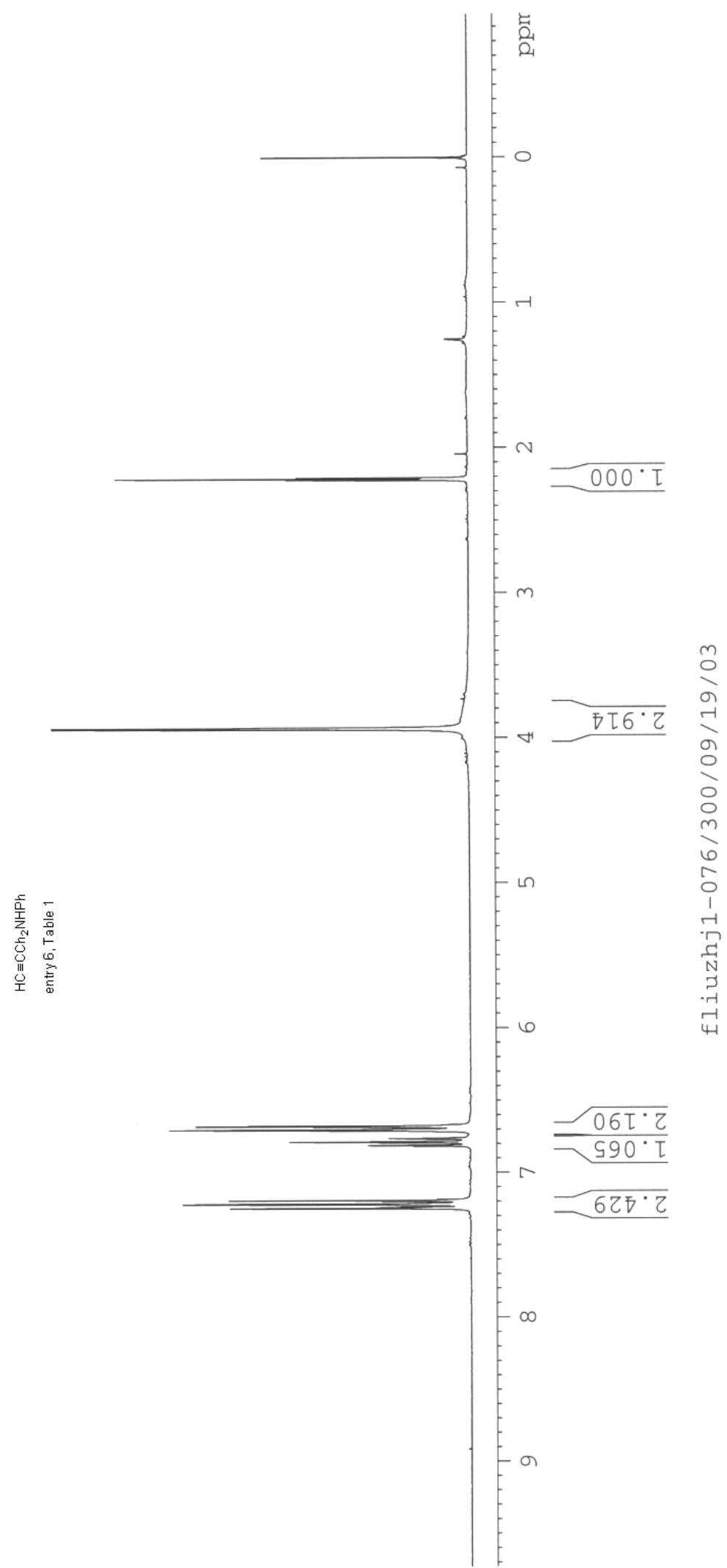




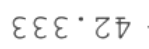

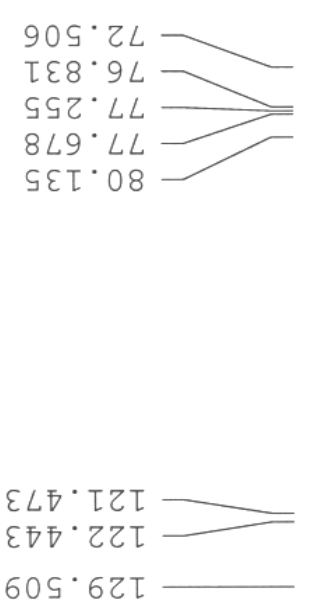

T29 $\angle \bar{~} \mathrm{~T}$

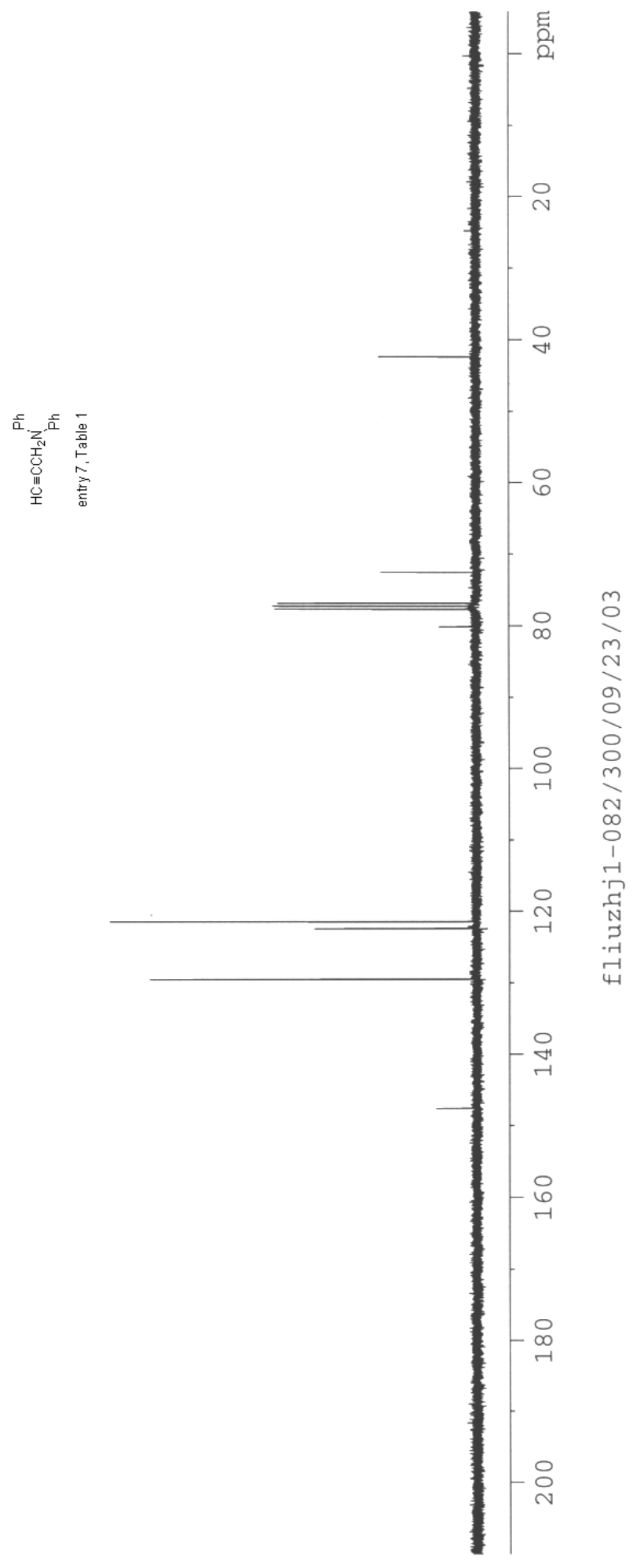

S13 

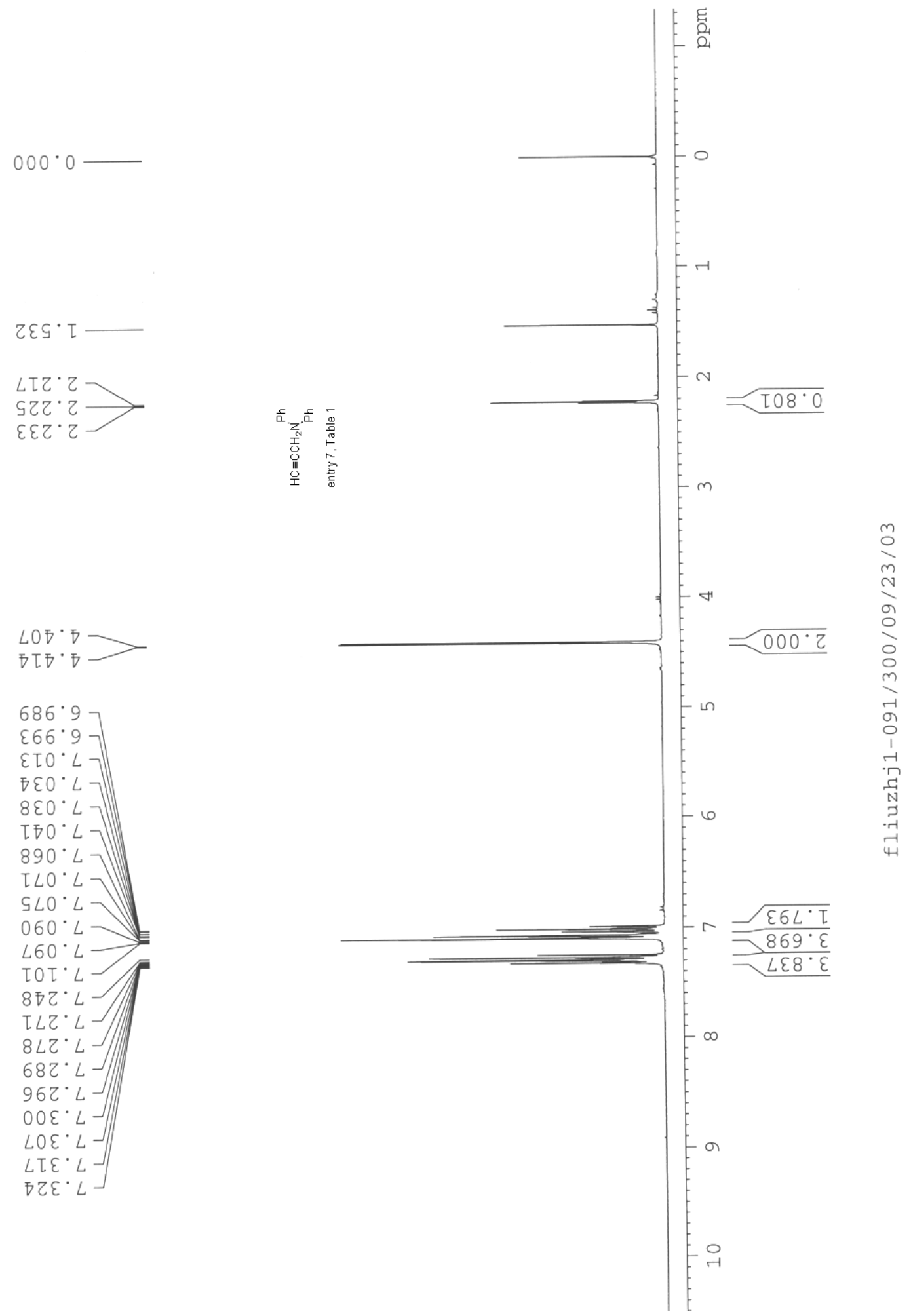


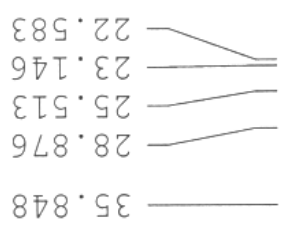

ISD ${ }^{\circ}$ IS

$\angle 78 \cdot 9 L$ $I L Z^{\circ} L L$
$\nabla 69^{\circ} L L$
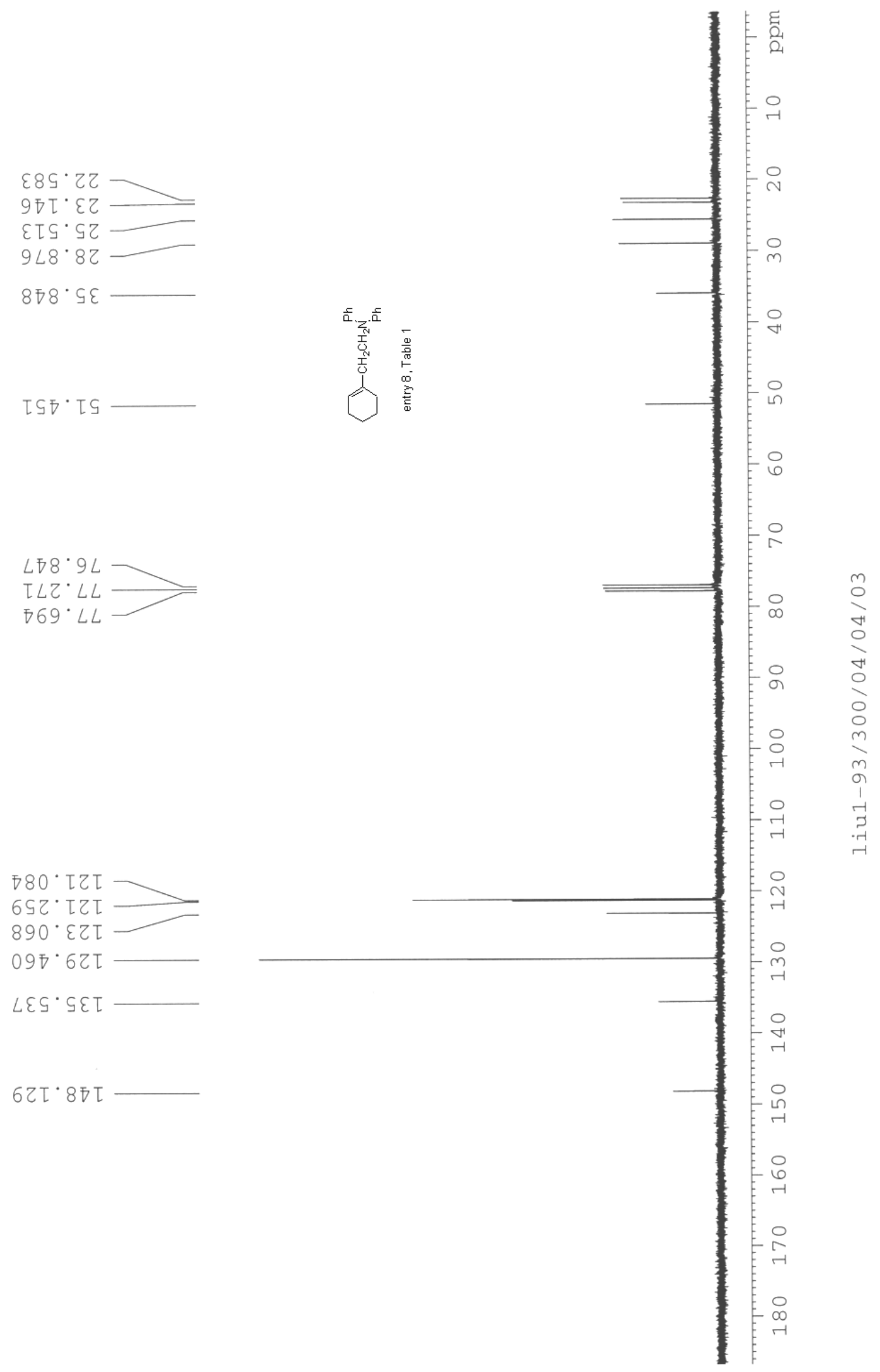

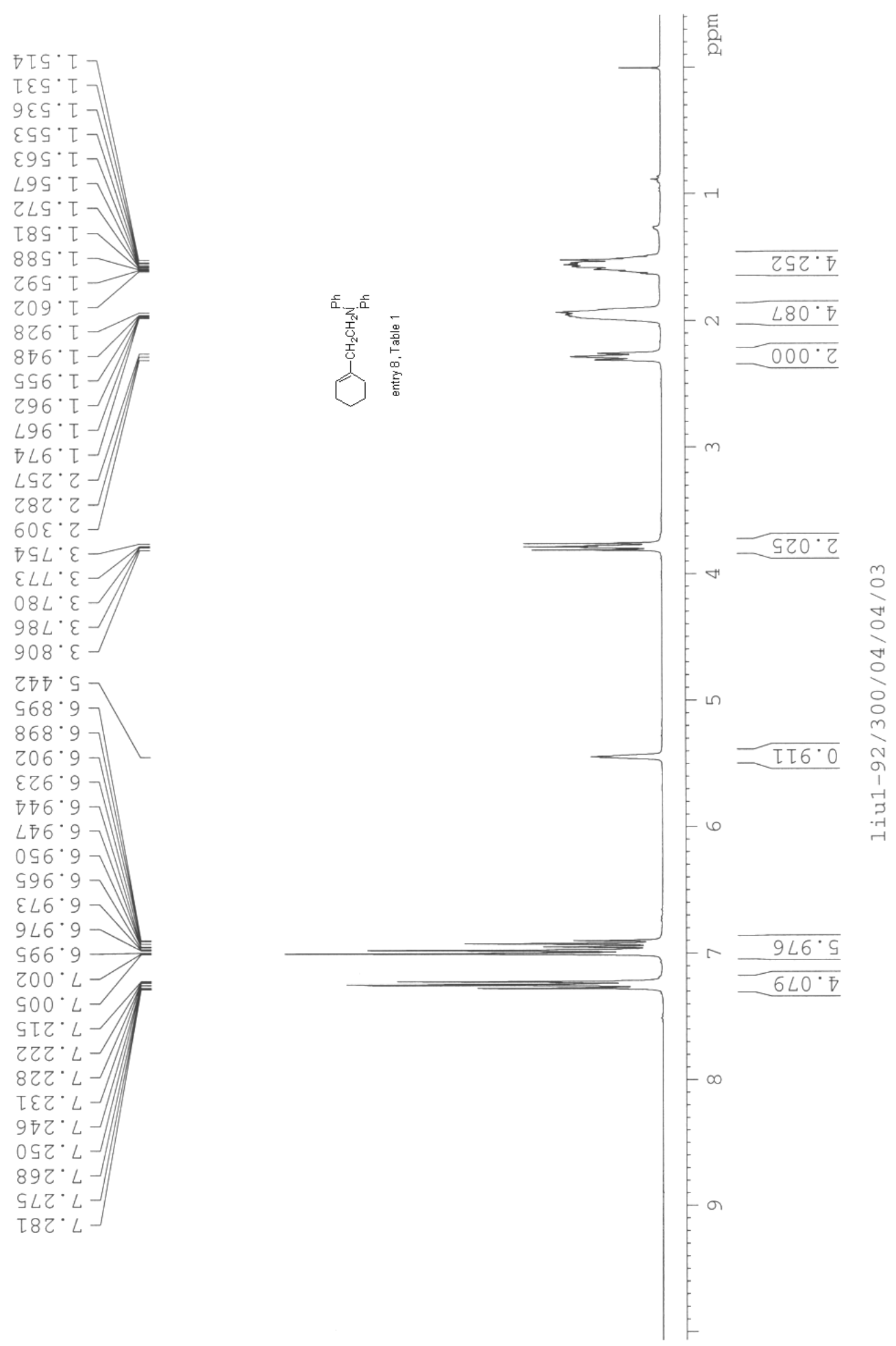

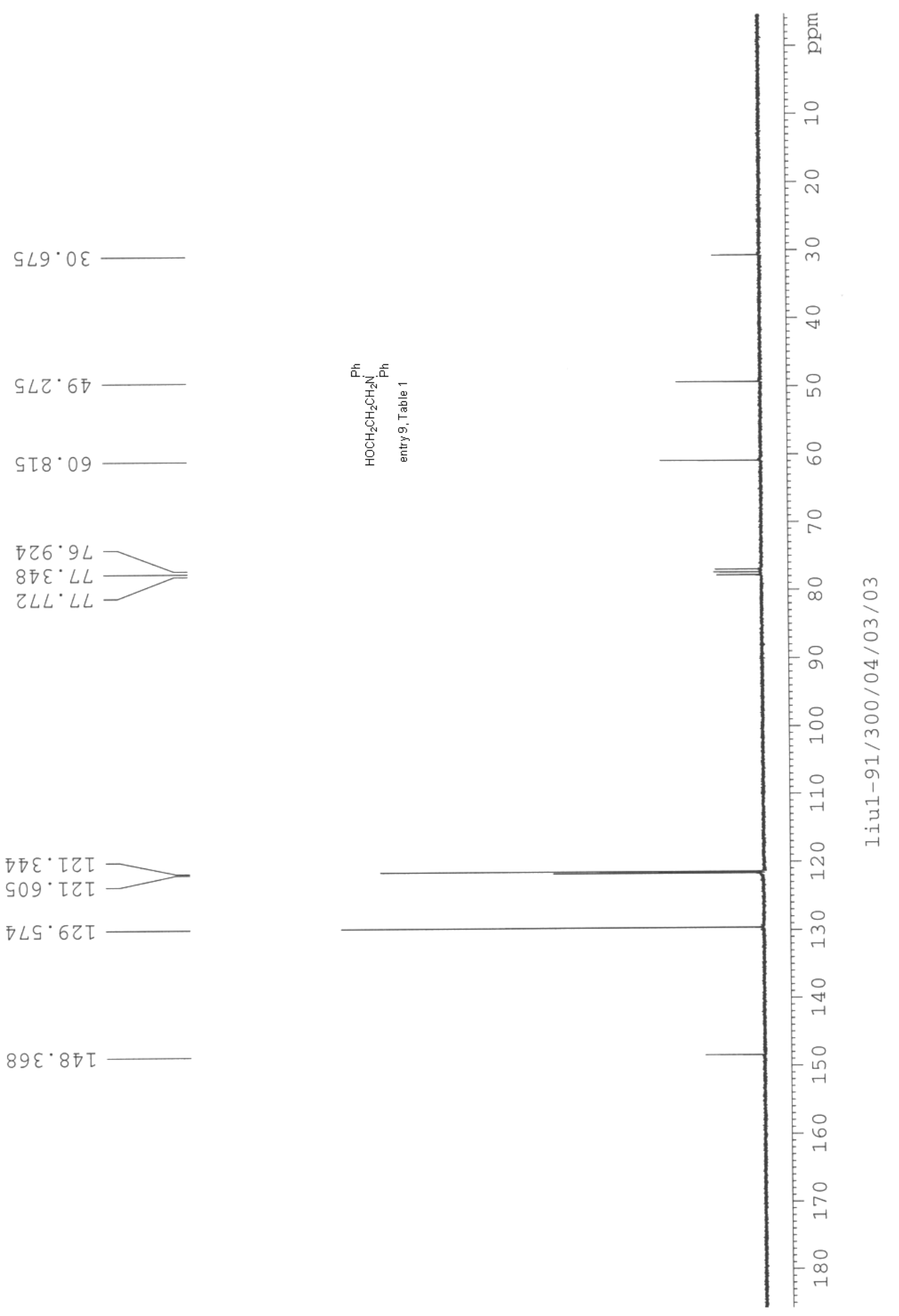
$000 \cdot 0$
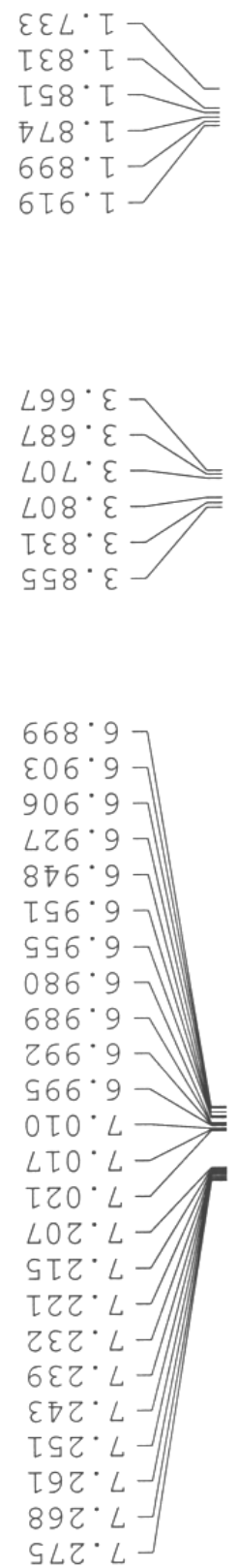

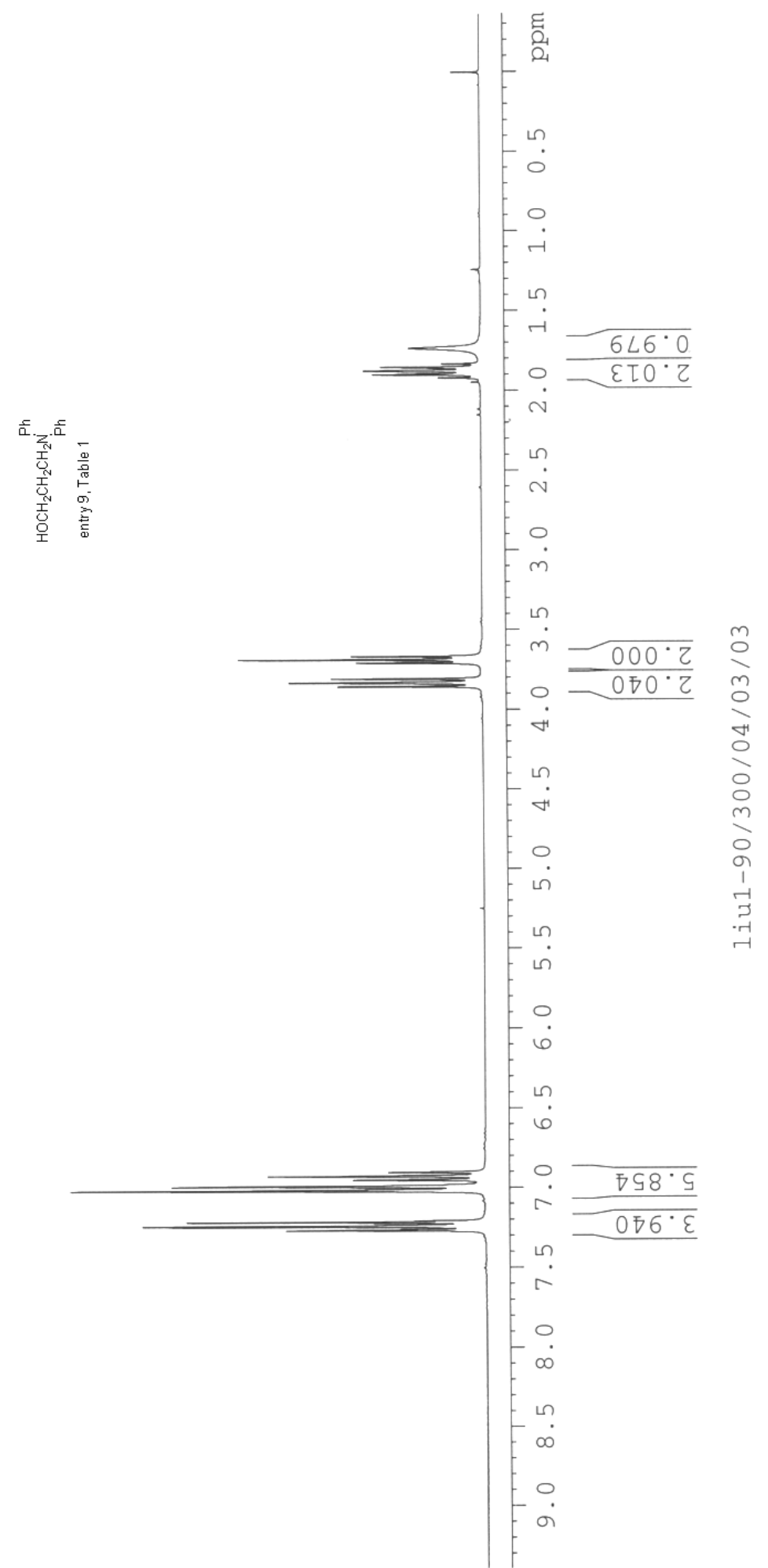




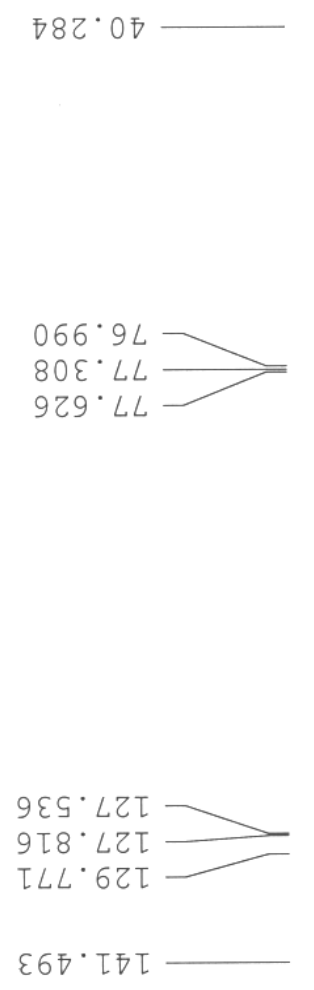

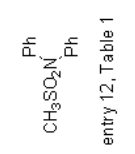

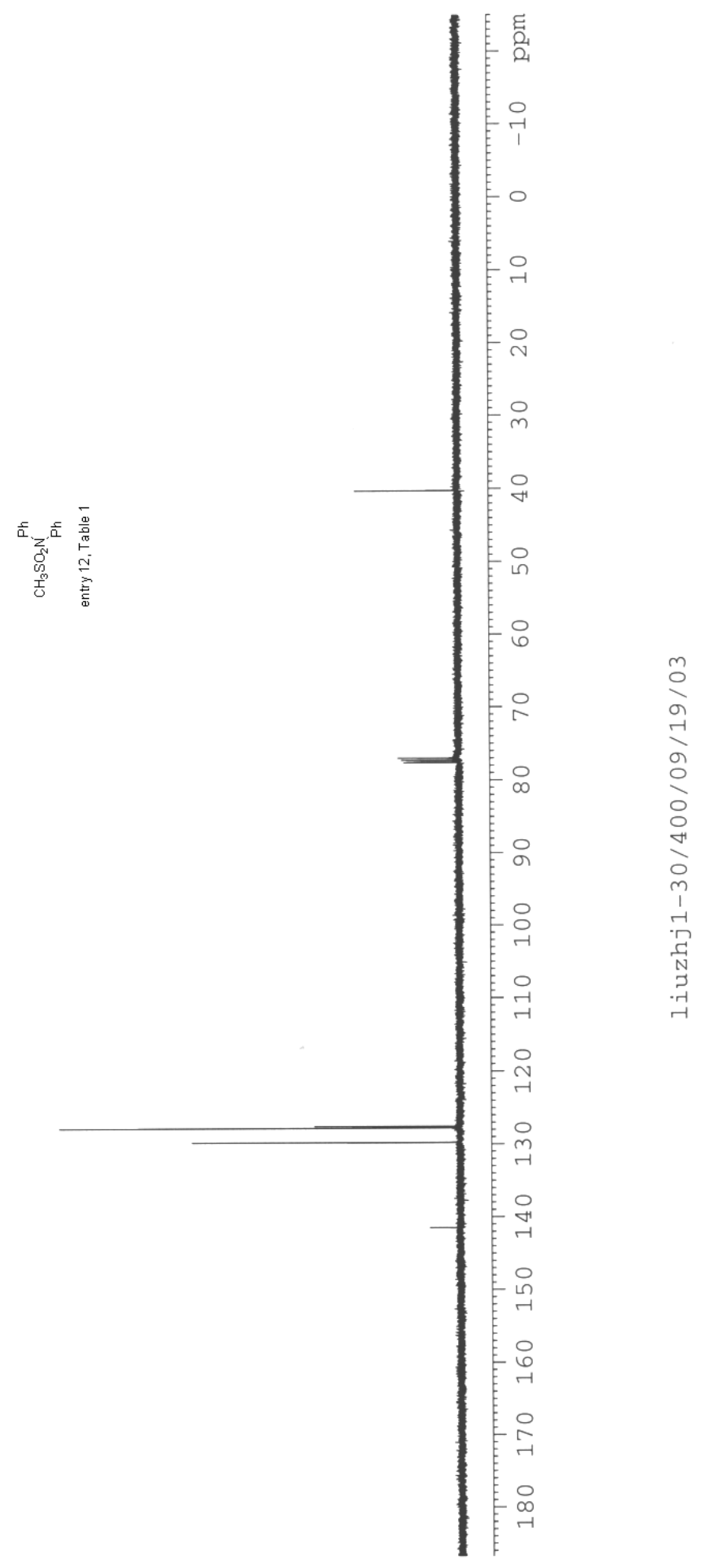



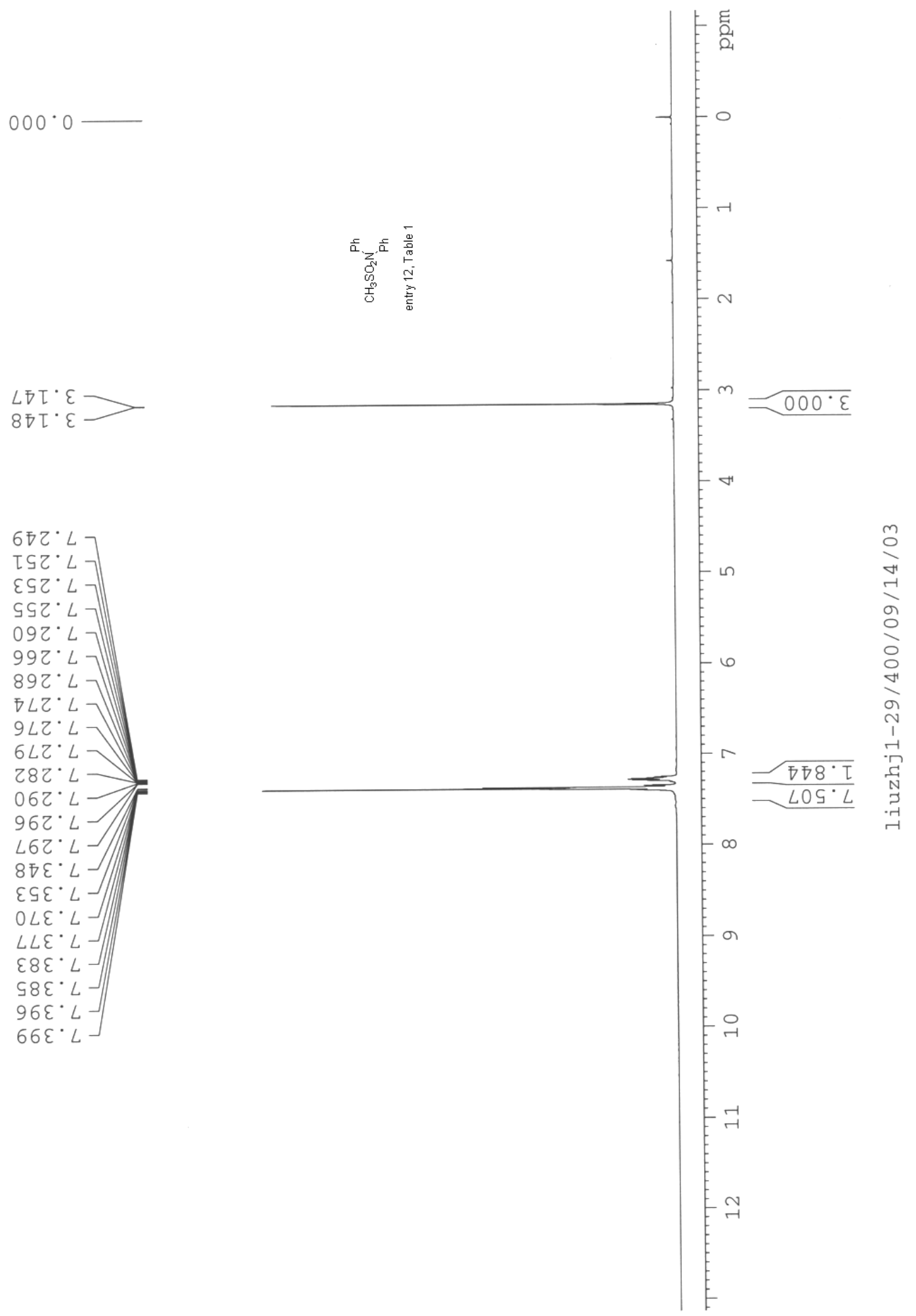


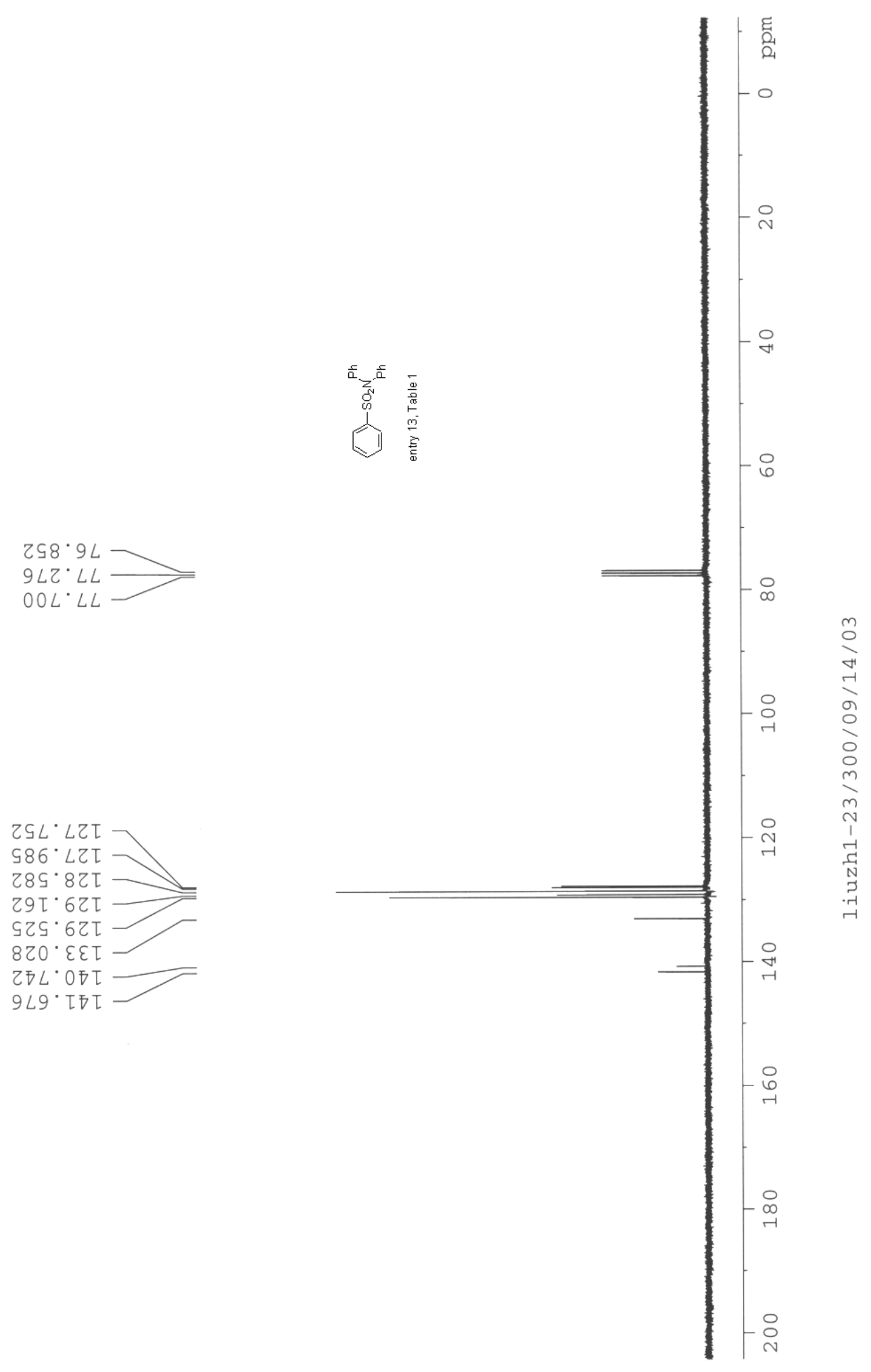


Ђध乙 $L$

бह乙 ${ }^{\circ} L$

8ठて $\cdot L$

$092^{\circ} L$

$\angle 97^{\circ} L$

$\nabla L Z^{\circ} L$

$062^{\circ} L$

०० $\varepsilon^{\circ} L$

LIE ${ }^{\circ}$

9 乙 $\varepsilon^{*} L$

$\varepsilon \varpi \varepsilon \cdot L$

$6 \nabla \varepsilon \cdot L$

งธ๋

6LF'

SOS $L$

$999^{\circ} L$

$O L S^{\circ} L$

$\nabla L G^{\circ} L-$

$969^{\circ} L$

$20 L^{\circ} L$

$\angle O L L^{\circ} L$

$\varepsilon\left[L^{\circ} L-\right.$

โEL $L^{\circ} L$

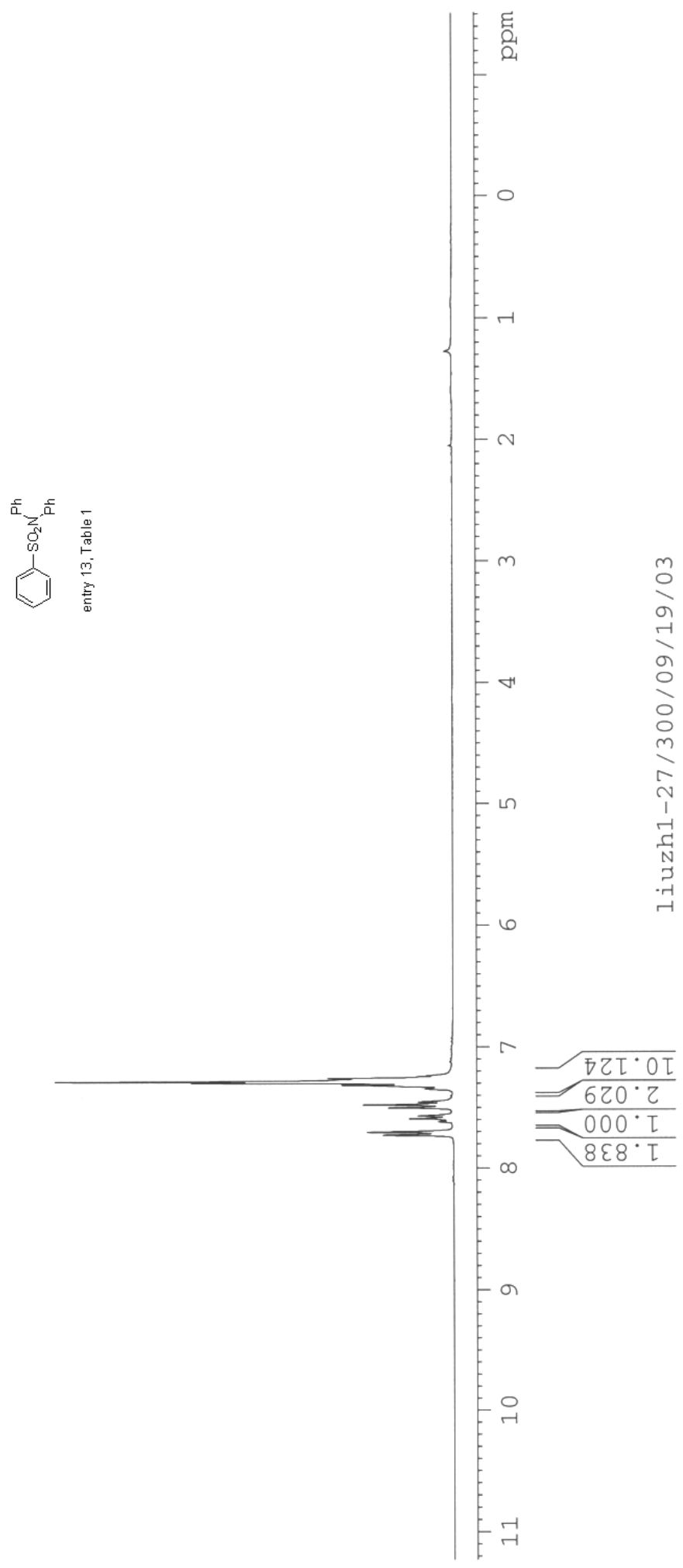



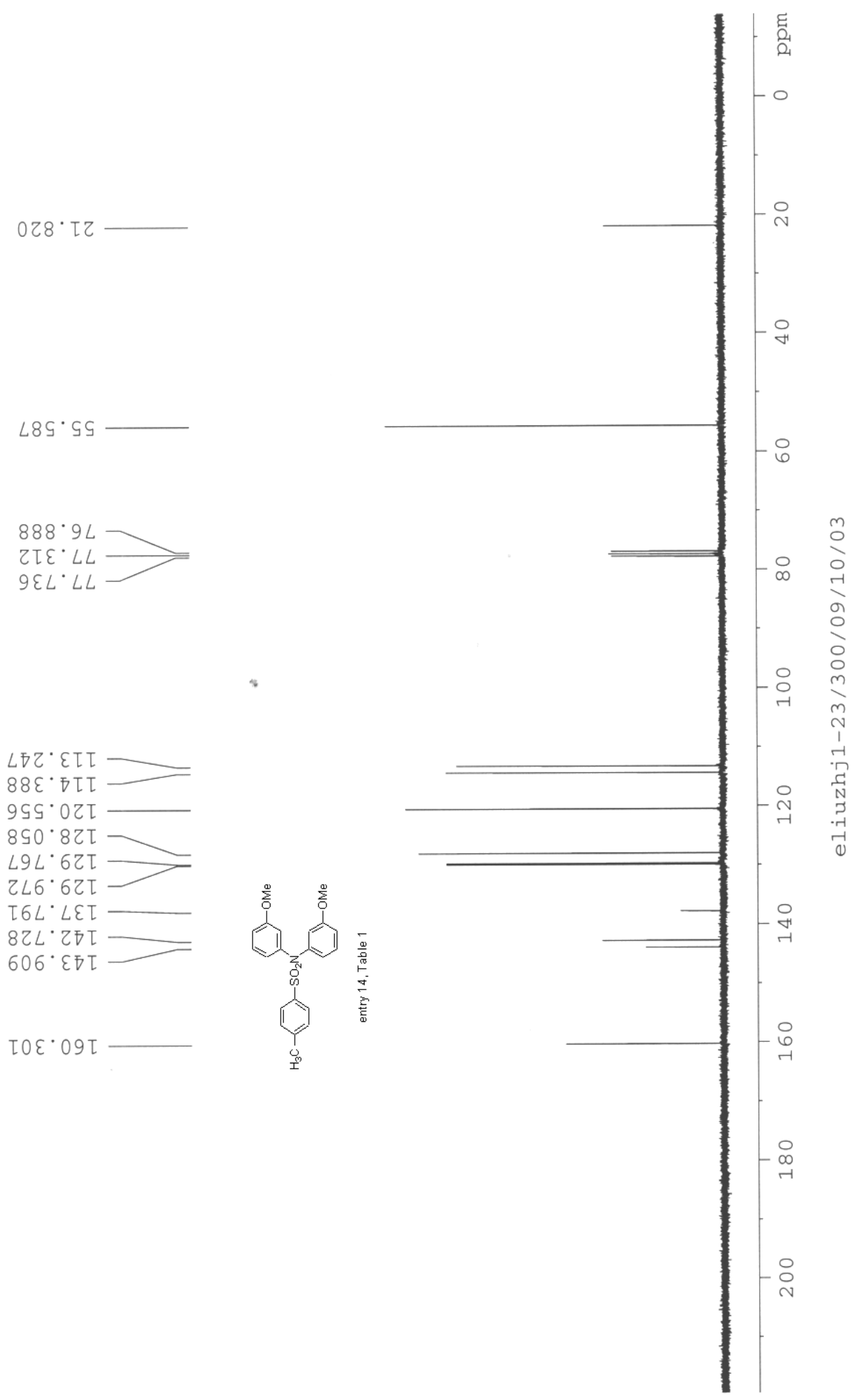


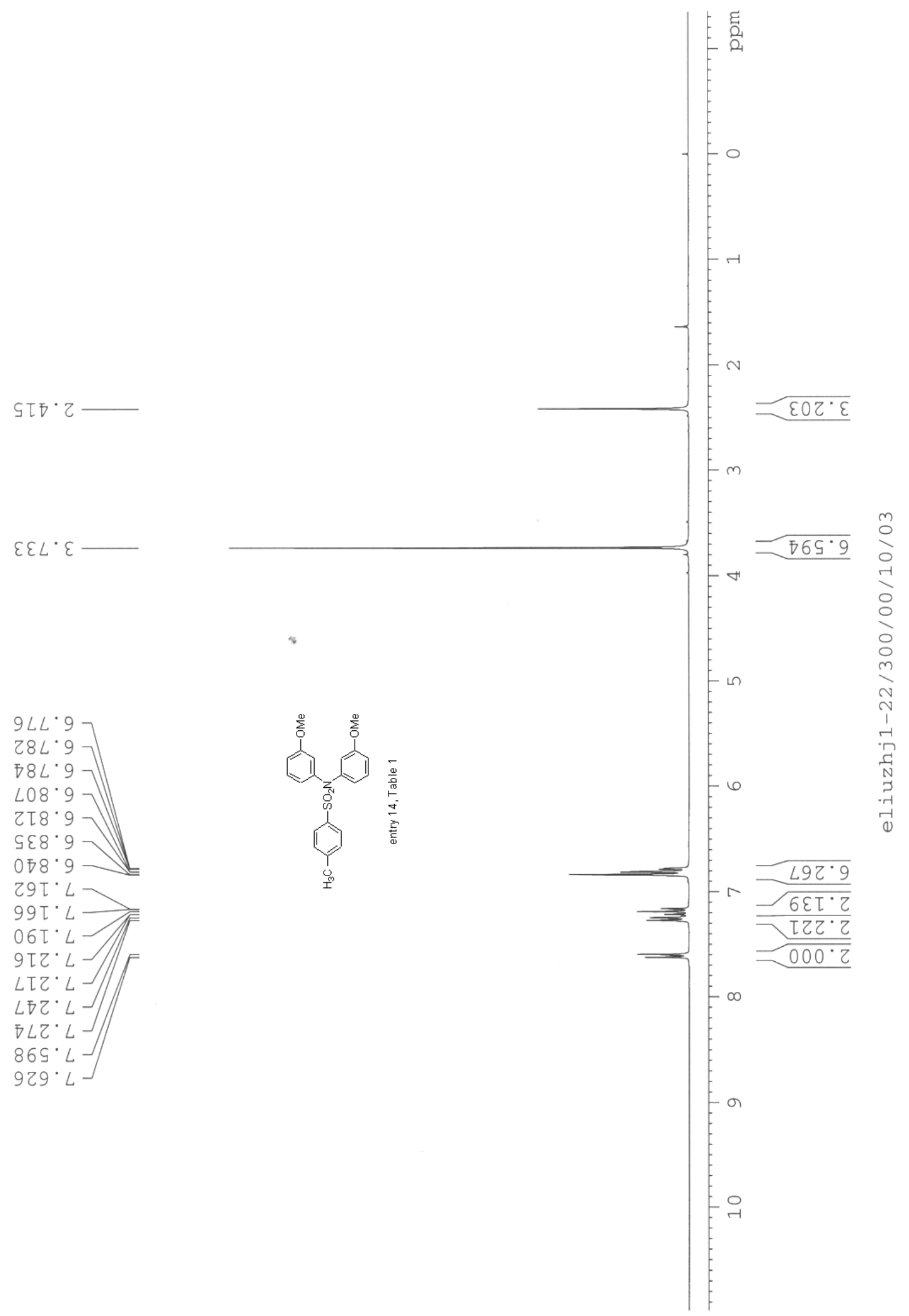



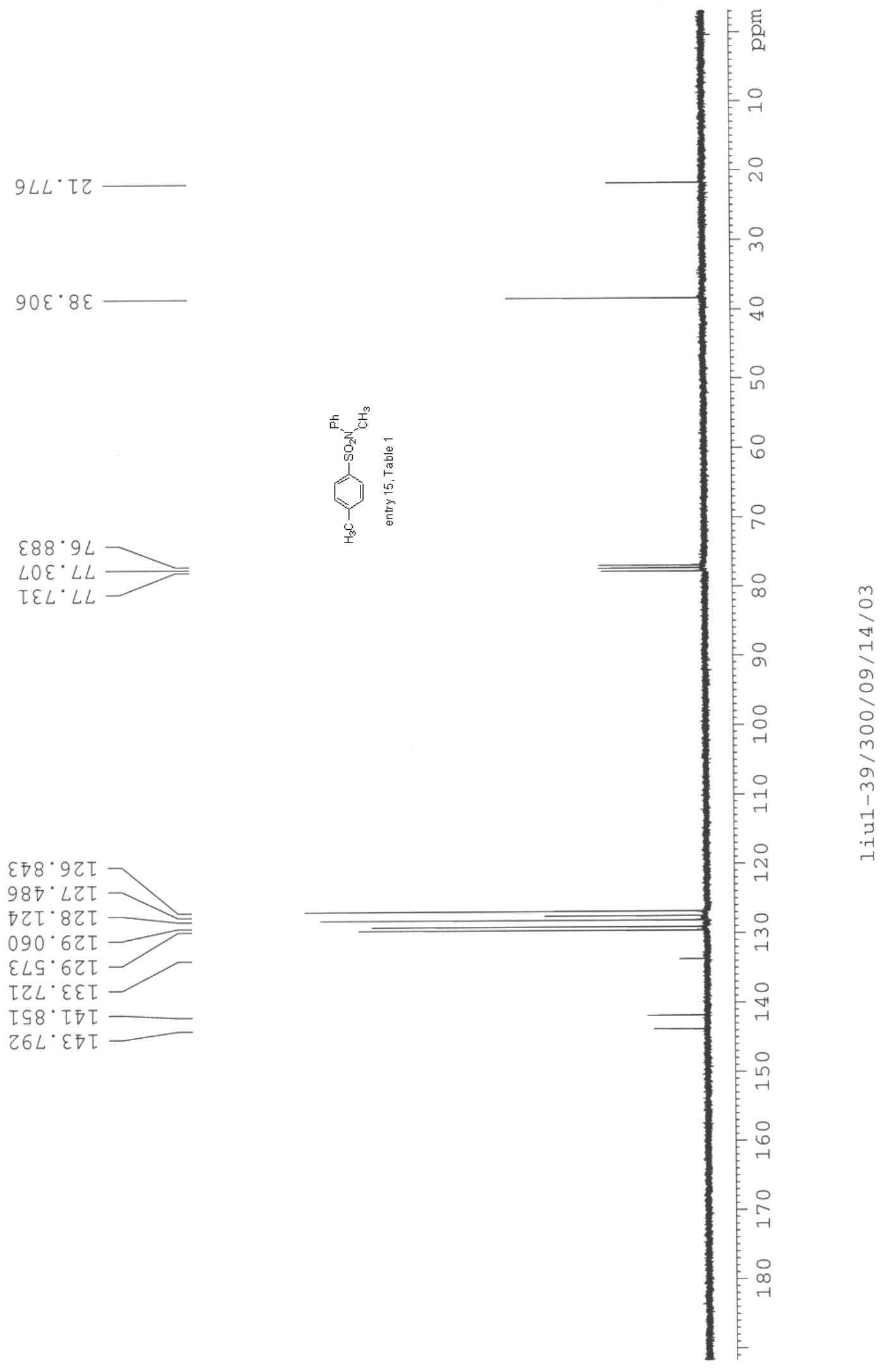

3
0
4
-1
9
0
0
0
0
$m$
0
$m$
1
-1
7
-7
-1 


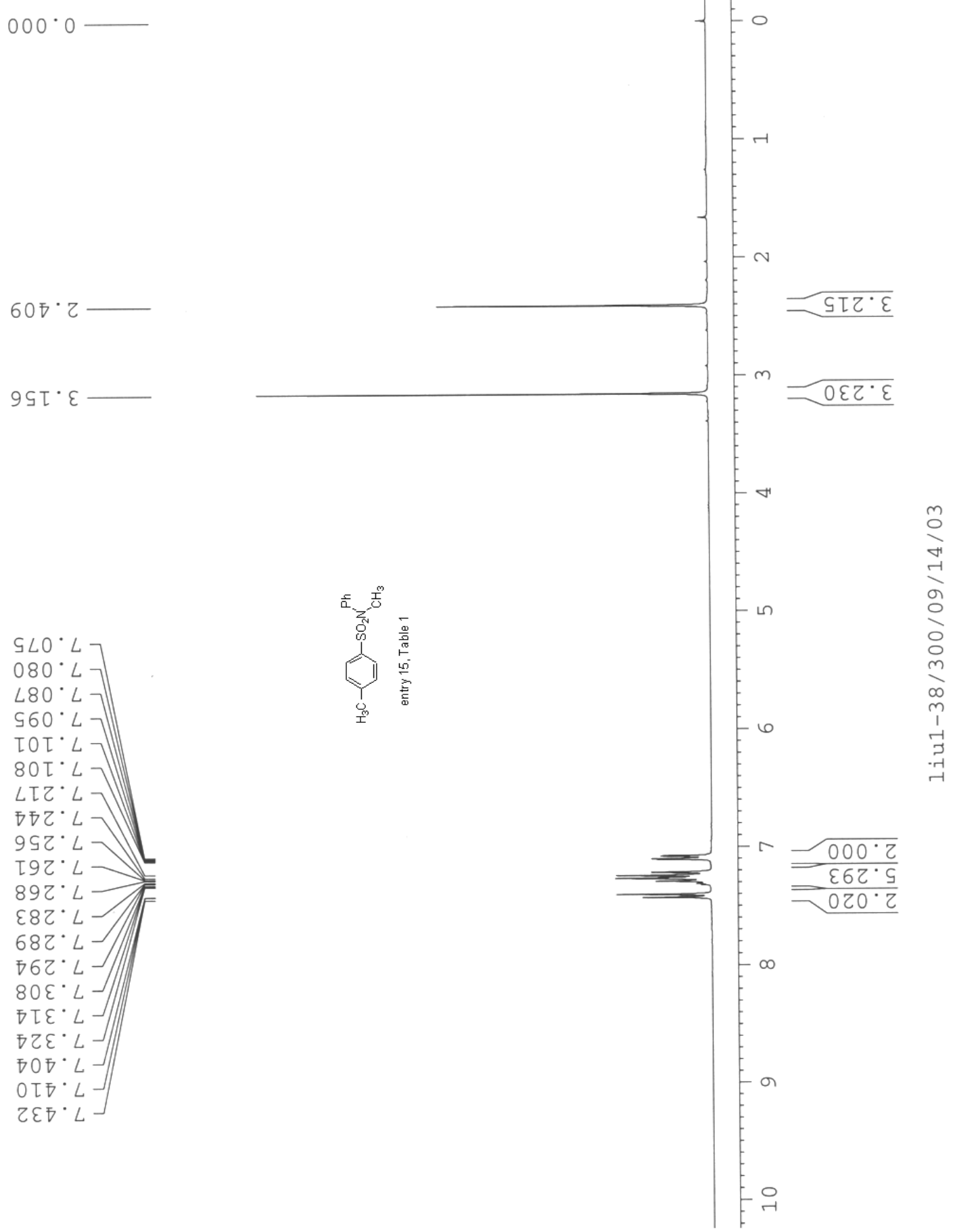

